\title{
THE NUMERICAL SOLUTION OF A NEUMANN PROBLEM FOR PARABOLIC SINGULARLY PERTURBED EQUATIONS WITH HIGH-ORDER TIME ACCURACY
}

\author{
P.W. HEMKER \\ CWI, Amsterdam, The Netherlands. \\ G.I. SHISHKIN \\ Institute of Mathematics and Mechanics, \\ Ural Branch of the Russian Academy of Science, Ekaterinburg, Russia. \\ L.P. SHISHKINA \\ Scientific Research Institute of Heavy Machine Building, Ekaterinburg, Russia.

\begin{abstract}
We study the discrete approximation of a Neumann problem on an interval for a singularly perturbed parabolic PDE. For this boundary value problem we construct a special piecewise-uniform mesh on which the discretization, based on the classical finite difference approximation, converges $\varepsilon$-uniformly with the order $\mathcal{O}\left(N^{-2} \ln ^{2} N+K^{-1}\right)$, where, respectively, $N$ and $K$ are the number of intervals in the space and the time mesh. With such discretizations we construct schemes of high order accuracy in the time. To obtain the better accuracy, we use a defect correction technique. Auxiliary discrete problems on the same time-mesh are introduced to correct the low order difference approximations. To validate the theoretical results, some numerical results for the new schemes are presented.
\end{abstract}

\section{Introduction}

For singularly perturbed boundary value problems, for parabolic PDEs with smooth data and without convection terms, a special discretization was studied in Shishkin cs $1,2,3,4,5$, for which the order of $\varepsilon$-uniform convergence is $\mathcal{O}\left(N^{-2} \ln ^{2} N+K^{-1}\right)$, where $N$ and $K$ denote, respectively, the number of intervals in the space and time discretization. For this discrete method the amount of computational work was primarily determined by the time discretization, which was of first order accuracy only. The improvement of the order of accuracy in time, maintaining $\varepsilon$-uniform convergence, by means of a defect correction technique was studied in Hemker et al ${ }^{6}$ for a Dirichlet problem and it could be achieved without essentially increasing the amount of computational work.

In this paper we show that we can extend the method for a Neumann problem, still obtaining the higher ( 2 nd or $3 \mathrm{rd}$ ) order of accuracy for the time variable and the second (except for a logarithmic factor) order accuracy in space. 


\section{The class of boundary value problems studied}

a) On the domain $G=D \times(0, T], D=(0,1)$ with the boundary $S=\bar{G} \backslash G$ we consider the singularly perturbed parabolic equation with Neumann boundary condition:

$$
\begin{aligned}
& L_{(1)} u(x, t) \equiv \varepsilon^{2} \frac{\partial}{\partial x}\left(a(x, t) \frac{\partial}{\partial x} u(x, t)\right)-c(x, t) u(x, t)- \\
&-p(x, t) \frac{\partial}{\partial t} u(x, t)=f(x, t), \quad(x, t) \in G, \quad \varepsilon \in(0,1], \\
& l_{(1)} u(x, t) \equiv \varepsilon \frac{\partial}{\partial n} u(x, t)=\psi(x, t), \quad(x, t) \in S_{1}, \\
& u(x, t)=\varphi(x, t), \quad(x, t) \in S_{0} .
\end{aligned}
$$

Here $^{a} S=S_{0} \cup S_{1}, S_{1}=\{(x, t): x=0$ or $x=1,0<t \leq T\}, S_{0}=\{(x, t):$ $x \in[0,1], t=0\}, \partial / \partial n$ is the derivative w.r.t. the outward bound normal to $S_{1}$. In $(1) a(x, t), c(x, t), p(x, t), f(x, t),(x, t) \in \bar{G}$, and $\varphi(x, t),(x, t) \in S_{0}$, $\psi(x, t),(x, t) \in S_{1}$ are sufficiently smooth and bounded functions

$$
0<a_{0} \leq a(x, t), \quad 0<p_{0} \leq p(x, t), \quad c(x, t) \geq 0, \quad(x, t) \in \bar{G} .
$$

When the parameter $\varepsilon$ tends to zero, in a neighborhood of the lateral boundary $S_{1}$ layers appear in the solution, which are described by an equation of parabolic type (parabolic boundary layers).

b) We assume that compatibility conditions which ensure sufficient smoothness of the problem solution are satisfied on the set $S_{0} \cap \overline{S_{1}}$. Then, for the solution of the problem and its components from the representation, we may write

$$
u(x, t)=U(x, t)+W(x, t), \quad(x, t) \in \bar{G},
$$

where $U(x, t)$ represents the regular part and $W(x, t)$ the singular part. For these components the following estimates are valid

$$
\begin{gathered}
\left|\frac{\partial^{k+k_{0}}}{\partial x^{k} \partial t^{k_{0}}} u(x, t)\right| \leq M \varepsilon^{-k}, \quad\left|\frac{\partial^{k+k_{0}}}{\partial x^{k} \partial t^{k_{0}}} U(x, t)\right| \leq M, \\
\left|\frac{\partial^{k+k_{0}}}{\partial x^{k} \partial t^{k_{0}}} W(x, t)\right| \leq M \varepsilon^{-k} \exp \left(-m \varepsilon^{-1} r(x, \Gamma)\right), \\
(x, t) \in \bar{G}, \quad k+2 k_{0} \leq 2 n+4,
\end{gathered}
$$

\footnotetext{
a The subscript (1) denotes that the symbol is introduced in equation (1).
} 
where $r(x, \Gamma)$ is the distance between the point $x \in \bar{D}$ and the set $\Gamma=\bar{D} \backslash D$.

Here and in the text below we denote by $M$ (or $m$ ) sufficiently large (or small) positive constants which do not depend on the value of parameter $\varepsilon$ or on the difference operators used.

\section{The finite difference schemes}

a) To solve problem (1) we first consider a classical finite difference method. On the set $\bar{G}$ we introduce the rectangular mesh

$$
\bar{G}_{h}=\bar{\omega} \times \bar{\omega}_{0},
$$

where $\bar{\omega}$ is a (possibly) non-uniform mesh of nodal points, $x^{i}$, in $[0,1], \bar{\omega}_{0}$ is a uniform mesh on the interval $[0, T] ; N$ and $K$ are the numbers of intervals in the grids $\bar{\omega}$ and $\bar{\omega}_{0}$ respectively. We define $\tau=T / K, h^{i}=x^{i+1}-x^{i}$, $h=\max _{i} h^{i}, h \leq M / N, G_{h}=G \cap \bar{G}_{h}, S_{h}=S \cap \bar{G}_{h}$.

For problem (1) we use the difference scheme

$$
\begin{gathered}
\Lambda_{(4)} z(x, t)=f(x, t), \quad(x, t) \in G_{h}, \\
\lambda_{(4)} z(x, t)=\psi^{h}(x, t), \quad(x, t) \in S_{1 h}, \quad z(x, t)=\varphi(x, t), \quad(x, t) \in S_{0 h} .
\end{gathered}
$$

Here

$$
\begin{aligned}
& \Lambda_{(4)} z(x, t) \equiv \varepsilon^{2} \delta_{\widehat{x}}\left(a^{h}(x, t) \delta_{\bar{x}} z(x, t)\right)-c(x, t) z(x, t)-p(x, t) \delta_{\bar{t}^{2}} z(x, t) ; \\
& \lambda_{(4)} z(x, t) \equiv-\varepsilon \delta_{x} z(x, t)+\frac{x^{i+1}-x^{i}}{2 \varepsilon a+h(x, t)}\left[c(x, t) z(x, t)+p(x, t) \delta_{\bar{t}} z(x, t)\right] \text {, } \\
& \psi^{h}(x, t)=a(x, t)\left(a^{+h}(x, t)\right)^{-1} \psi(x, t)-\frac{x^{i+1}-x^{i}}{2 \varepsilon a^{+h}(x, t)} f(x, t), \quad x=x^{i}=0 ; \\
& \lambda_{(4)} z(x, t) \equiv \varepsilon \delta_{\bar{x}} z(x, t)+\frac{x^{2}-x^{i-1}}{2 \varepsilon a^{h}(x, t)}\left[c(x, t) z(x, t)+p(x, t) \delta_{\bar{t}} z(x, t)\right], \\
& \psi^{h}(x, t)=a(x, t)\left(a^{h}(x, t)\right)^{-1} \psi(x, t)-\frac{x^{i}-x^{i-1}}{2 \varepsilon a^{h}(x, t)} f(x, t), \quad x=x^{i}=1 ; \\
& \delta_{\widehat{x}}\left(a^{h}\left(x^{2}, t\right) \delta_{\bar{x}} z\left(x^{2}, t\right)\right)=2\left(x^{2+1}-x^{2-1}\right)^{-1}\left(a^{+h}\left(x^{2}, t\right) \delta_{x} z\left(x^{i}, t\right)-a^{h}\left(x^{i}, t\right) \delta_{\bar{x}} z\left(x^{2}, t\right)\right), \\
& a^{h}\left(x^{2}, t\right)=a\left(\left(x^{i-1}+x^{i}\right) / 2, t\right), \quad a^{+h}\left(x^{i}, t\right)=a^{h}\left(x^{i+1}, t\right)=a\left(\left(x^{i}+x^{i+1}\right) / 2, t\right),
\end{aligned}
$$

$\delta_{x} z(x, t)$ and $\delta_{\bar{x}} z(x, t), \delta_{\bar{t}} z(x, t)$ are the forward and backward differences, and the difference operator $\delta_{\widehat{x}}\left(a^{h}(x, t) \delta_{\bar{x}^{x}}(x, t)\right)$ is an approximation of the operator $\frac{\partial}{\partial x}\left(a(x, t) \frac{\partial}{\partial x} u(x, t)\right)$ on the non-uniform mesh.

Taking into account estimates of the derivatives we find that the solution of the difference scheme (4), (3) converges for a fixed value of the parameter $\varepsilon$ :

$$
|u(x, t)-z(x, t)| \leq M\left(\varepsilon^{-1} N^{-1}+\tau\right), \quad(x, t) \in \bar{G}_{h} .
$$


b) Let us construct the scheme, convergent $\varepsilon$-uniformly. On $\bar{G}$ we the mesn

$$
\bar{G}_{h}^{*}=\bar{\omega}^{*}(\sigma) \times \bar{\omega}_{0}
$$

where $\bar{\omega}_{0}=\bar{\omega}_{0(3)}$ and $\bar{\omega}^{*}=\bar{\omega}^{*}(\sigma)$ is a special precewise uniform pending on the parameter $\sigma \in \mathbb{R}, \sigma=\sigma_{(6)}(\varepsilon, N)=\min [d / 4, m \varepsilon \ln$. $m=m_{(6)}$ is an arbitrary positive number. The mesh $\bar{\omega}^{*}(\sigma)$ is cons follows. The interval $[0,1]$ is divided in three parts $[0, \sigma],[\sigma, 1-\sigma]$, $0<\sigma \leq 1 / 4$. In each part we use a uniform mesh, with $N / 2$ subir $[\sigma, 1-\bar{\sigma}]$ and with $N / 4$ subintervals in each interval $[0, \sigma]$ and [ 1 Theorem 3.1 Let the estimate (2) hold for the solution of (1). solution of (4), (6) converges $\varepsilon$-uniformly to the solution of (1) with bounds

$$
|u(x, t)-z(x, t)| \leq M\left(N^{-2} \ln ^{2} N+\tau\right), \quad(x, t) \in \bar{G}_{h}^{*} .
$$

\section{Numerical results}

To see the effect of the special mesh in practice, we take the model

$$
\begin{gathered}
L_{(8)} u(x, t) \equiv \varepsilon^{2} \frac{\partial^{2}}{\partial x^{2}} u(x, t)-\frac{\partial}{\partial t} u(x, t)=f(x, t), \quad(x, t) \in G ; \\
\varepsilon \frac{\partial}{\partial n} u(x, t)=\psi(x, t), \quad(x, t) \in S_{1}, \\
u(x, t)=\varphi(x, t), \quad(x, t) \in S_{0},
\end{gathered}
$$

where

$$
\begin{gathered}
f(x, t)=-4 t^{3}, \quad(x, t) \in \bar{G} \\
\psi(0, t)=\psi(1, t)=-(128 / 35) \pi^{-1 / 2} t^{7 / 2}, \quad 0<t \leq T, \quad T=1 . \\
\varphi(x, t)=0, \quad(x, t) \in S_{0} .
\end{gathered}
$$

We present the error $E(N, K, \varepsilon)$, defined by

$$
E(N, K, \varepsilon)=\max _{(x, t) \in \bar{G}_{h}}\left|z(x, t)-u^{*}(x, t)\right| .
$$

Here $u^{*}(x, t)$ is the piecewise linear interpolation obtained from the $\mathrm{r}$ solution $z(x, t)$ on an adapted fine mesh (6) with parameters $m=2$, $N^{*}>N, K^{*}>>K$.

In Table 1 we give the results for the scheme (4), (6) with $K$ Here we can clearly see that, in accordance with estimate (7), the convergence is $\mathcal{O}\left(N^{-2} \ln ^{2} N+K^{-1}\right)$ for large $N, K$. 


\begin{tabular}{|c|cccccc|}
\hline$\varepsilon \backslash N$ & 8 & 16 & 32 & 64 & 128 & 256 \\
\hline 1.0 & $1.065 \mathrm{e}-1$ & $4.473 \mathrm{e}-2$ & $1.971 \mathrm{e}-2$ & $8.740 \mathrm{e}-3$ & $3.645 \mathrm{e}-3$ & $1.199 \mathrm{e}-3$ \\
$2^{-1}$ & $3.065 \mathrm{e}-1$ & $1.358 \mathrm{e}-1$ & $6.185 \mathrm{e}-2$ & $2.794 \mathrm{e}-2$ & $1.177 \mathrm{e}-2$ & $3.891 \mathrm{e}-3$ \\
$2^{-2}$ & $6.723 \mathrm{e}-1$ & $2.922 \mathrm{e}-1$ & $1.315 \mathrm{e}-1$ & $5.905 \mathrm{e}-2$ & $2.480 \mathrm{e}-2$ & $8.184 \mathrm{e}-3$ \\
$2^{-3}$ & $1.485 \mathrm{e}+0$ & $6.305 \mathrm{e}-1$ & $2.755 \mathrm{e}-1$ & $1.212 \mathrm{e}-1$ & $5.036 \mathrm{e}-2$ & $1.652 \mathrm{e}-2$ \\
$2^{-4}$ & $3.352 \mathrm{e}+0$ & $1.436 \mathrm{e}+0$ & $6.054 \mathrm{e}-1$ & $2.568 \mathrm{e}-1$ & $1.042 \mathrm{e}-1$ & $3.374 \mathrm{e}-2$ \\
$2^{-5}$ & $3.474 \mathrm{e}+0$ & $2.108 \mathrm{e}+0$ & $1.161 \mathrm{e}+0$ & $5.696 \mathrm{e}-1$ & $2.225 \mathrm{e}-1$ & $7.035 \mathrm{e}-2$ \\
$2^{-6}$ & $3.435 \mathrm{e}+0$ & $2.069 \mathrm{e}+0$ & $1.226 \mathrm{e}+0$ & $5.604 \mathrm{e}-1$ & $2.529 \mathrm{e}-1$ & $8.844 \mathrm{e}-2$ \\
$2^{-12}$ & $3.435 \mathrm{e}+0$ & $2.069 \mathrm{e}+0$ & $1.226 \mathrm{e}+0$ & $5.604 \mathrm{e}-1$ & $2.529 \mathrm{e}-1$ & $8.844 \mathrm{e}-2$ \\
\hline $\bar{E}(N)$ & $3.474 \mathrm{e}+00$ & $2.108 \mathrm{e}+00$ & $1.226 \mathrm{e}+00$ & $5.696 \mathrm{e}-01$ & $2.529 \mathrm{e}-1$ & $8.844 \mathrm{e}-2$ \\
\hline
\end{tabular}

Table 1: Errors $E\left(N, N^{2}, \varepsilon\right)$ for the special method (4), (6).

In this table the function $E\left(N, N^{2}, \varepsilon\right)$ is defined by (9) for $N^{*}=512, K^{*}=512^{2}$.

\section{Improved time-accuracy. A scheme based on defect correction}

a) In this section we construct a new discrete method based on defect correction, which also converges $\varepsilon$-uniformly to the solution of the boundary value problem, but with an order of accuracy (w.r.t. the timestep $\tau$ ) higher than in (7).

The idea is similar to the one considered in Hemker et al ${ }^{6}$ for the Dirichlet problem. For the difference scheme $(4),(6)$ the error in the approximation of the partial derivative $(\partial / \partial t) u(x, t)$ is caused by the divided difference $\delta_{\bar{t}} z(x, t)$ and is associated with the truncation error given by the relation

$$
\frac{\partial}{\partial t} u(x, t)-\delta_{\bar{t}} u(x, t)=2^{-1} \tau \frac{\partial^{2}}{\partial t^{2}} u(x, t)-6^{-1} \tau^{2} \frac{\partial^{3}}{\partial t^{3}} u(x, t-\vartheta),
$$

where $\vartheta \in[0, \tau]$. Therefore we now use for the approximation of $(\partial / \partial t) u(x, t)$ the expression

$$
\delta_{\bar{t}} u(x, t)+\tau \delta_{\bar{t} \bar{t}} u(x, t) / 2,
$$

where $\delta_{\bar{t} \bar{t}} u(x, t) \equiv \delta_{t \bar{t}} u(x, t-\tau), \quad \delta_{t \bar{t}} u(x, t)$ is the second central divided difference. We can evaluate a better approximation than (4a) by defect correction

$$
\Lambda_{(4)} z^{c}(x, t)=f(x, t)+2^{-1} p(x, t) \tau \frac{\partial^{2}}{\partial t^{2}} u(x, t),
$$

$\tau$ is step-size of the mesh $\bar{\omega}_{0} ; z^{c}(x, t)$ is the "corrected" solution. Instead of $\left(\partial^{2} / \partial t^{2}\right) u(x, t)$ we shall use $\delta_{\bar{t} \bar{t}} z(x, t)$, where $z(x, t),(x, t) \in G_{h(6)}$ is the solution of the difference scheme $(4),(6)$. The new solution $z^{c}(x, t)$ has an accuracy of $\mathcal{O}\left(\tau^{2}\right)$ w.r.t. the time variable.

When constructing the scheme with order of accuracy in time greater than two, in the case of the Neumann problem it -in addition- is necessary to make a correction in the difference derivative $\delta_{\bar{t}} z(x, t)$ in the boundary condition. 
b) Let us first consider the modified difference scheme with of second order accuracy in $\tau$. We denote by $\delta_{k \bar{t}} z(x, t)$ the backward difference of order $k$ :

$$
\begin{aligned}
& \delta_{k \bar{t}} z(x, t)=\left(\delta_{k-1 \bar{t}} z(x, t)-\delta_{k-1 \bar{t}} z(x, t-\tau)\right) / \tau, \\
& t \geq k \tau, \quad k \geq 1 ; \quad \delta_{0 \bar{t}} z(x, t)=z(x, t), \quad(x, t) \in \bar{G}_{h} .
\end{aligned}
$$

We consider the boundary value problem (1). When constructing difference schemes of second order accuracy in $\tau$ in (11), instead of $\left(\partial^{2} / \partial t^{2}\right) u(x, t)$ we use $\delta_{2,} z(x, t)$, which is the second divided difference of the solution to the discrete problem (4), (6). On the mesh $\bar{G}_{h}$ we consider the finite difference scheme (4), writing

$$
\begin{aligned}
\Lambda_{(4)} z^{(1)}(x, t) & =f(x, t), \quad(x, t) \in G_{h}, \\
\lambda_{(4)} z^{(1)}(x, t) & =\psi^{h}(x, t), \quad(x, t) \in S_{1 h} . \\
z^{(1)}(x, t) & =\varphi(x, t), \quad(x, t) \in S_{0 h} .
\end{aligned}
$$

Then for the boundary value problem (1) we now get the difference equation:

$$
\begin{gathered}
\Lambda_{(4)} z^{(2)}(x, t)=f(x, t)+\left\{\begin{array}{l}
p(x, t) 2^{-1} \tau \frac{\partial^{2}}{\partial t^{2}} u(x, 0), \quad t=\tau, \\
p(x, t) 2^{-1} \tau \delta_{2} z^{(1)}(x, t), \quad t \geq 2 \tau, \quad(x, t) \in G_{h},
\end{array}\right. \\
\lambda_{(4)} z^{(2)}(x, t)=\psi^{h}(x, t), \quad(x, t) \in S_{1 h}, \\
z^{(2)}(x, t)=\varphi(x, t), \quad(x, t) \in S_{0 h} .
\end{gathered}
$$

Here $z^{(1)}(x, t)$ is the solution of the discrete problem (12), (6), and the derivative $\frac{\partial^{2}}{\partial t^{2}} u(x, 0)$ is obtained from the equation (1a). We shall call $z^{(2)}(x, t)$ the solution of difference scheme (13), (12), (6) (or shortly, (13), (6)).

For simplicity, in the remainder of this section we take a homogeneous initial condition:

$$
\varphi(x, 0)=0, \quad x \in \bar{D} .
$$

Under the homogeneous initial condition (14), the following estimate holds for the solution of problem (13), (6)

$$
\left|u(x, t)-z^{(2)}(x, t)\right| \leq M\left[N^{-2} \ln ^{2} N+\tau^{2}\right], \quad(x, t) \in \bar{G}_{h} .
$$

Theorem 5.1 Let condition (14) hold and assume in equation (1) that a $\epsilon$ $H^{(\alpha+2 n+1)}(\bar{G}), c, p, f \in H^{(\alpha+2 n)}(\bar{G}), \varphi \in H^{(\alpha+2 n)}(\bar{G}), \alpha>4, n=1$ and let $\downarrow \in H^{(\alpha+2 n)}$ for $n=1$. Then for the solution of difference scheme (13), the estimate (15) holds. 
c) Analogously we construct a difference scheme with third order accuracy in $\tau$. For problem (1) on the mesh $\bar{G}_{h}$ we consider the difference scheme

$$
\begin{gathered}
\Lambda_{(4)} z^{(3)}(x, t)=f(x, t)+ \\
+\left\{\begin{array}{l}
p(x, t)\left(C_{11} \tau \frac{\partial^{2}}{\partial t^{2}} u(x, 0)+C_{12} \tau^{2} \frac{\partial^{3}}{\partial t^{3}} u(x, 0)\right), \quad t=\tau, \\
p(x, t)\left(C_{21} \tau \frac{\partial^{2}}{\partial t^{2}} u(x, 0)+C_{22} \tau^{2} \frac{\partial^{3}}{\partial t^{3}} u(x, 0)\right), \quad t=2 \tau, \\
p(x, t)\left(C_{31} \tau \delta_{2} z^{(2)}(x, t)+C_{32} \tau^{2} \delta_{3 \bar{t}} z^{(1)}(x, t)\right), t \geq 3 \tau, \quad(x, t) \in G_{h},
\end{array}\right. \\
\lambda_{(4)} z^{(3)}(x, t)=\psi^{h}(x, t)- \\
- \begin{cases}4^{-1} \varepsilon^{-1}\left(x^{i+1}-x^{i}\right) \tau\left(a^{+h}(x, t)\right)^{-1} p(x, t) \frac{\partial^{2}}{\partial t^{2}} u(x, 0), x=x^{i}=0, \\
4^{-1} \varepsilon^{-1}\left(x^{i}-x^{i-1}\right) \tau\left(a^{h}(x, t)\right)^{-1} p(x, t) \frac{\partial^{2}}{\partial t^{2}} u(x, 0), \quad x=x^{i}=1, t=\tau,\end{cases} \\
\lambda_{(4)} z^{(3)}(x, t)=\psi^{h}(x, t)-\quad(x, t) \in S_{1 h}, \\
-\left\{\begin{array}{cc}
4^{-1} \varepsilon^{-1}\left(x^{i+1}-x^{i}\right) \tau\left(a^{+h}(x, t)\right)^{-1} p(x, t) \delta_{2 \bar{t}^{2}} z^{(1)}(x, t), x=x^{i}=0, \\
4^{-1} \varepsilon^{-1}\left(x^{i}-x^{i-1}\right) \tau\left(a^{h}(x, t)\right)^{-1} p(x, t) \delta_{2 \bar{t}} z^{(1)}(x, t), & x=x^{i}=1, t \geq 2 \tau,
\end{array}\right. \\
z^{(3)}(x, t)=\varphi(x, t), \quad(x, t) \in S_{0 h} .
\end{gathered}
$$

Here $z^{(1)}(x, t)$ and $z^{(2)}(x, t)$ are the solutions of problems (12), (6) and (13), (6) respectively, the derivatives $\left(\partial^{2} / \partial t^{2}\right) u(x, 0),\left(\partial^{3} / \partial t^{3}\right) u(x, 0)$ are obtained from equation (la), the coefficients $C_{i j}$ are determined by

$$
C_{11}=C_{21}=C_{31}=1 / 2, \quad C_{12}=C_{32}=1 / 3, \quad C_{22}=5 / 6 .
$$

We shall call $z^{(3)}(x, t)$ the solution of the difference scheme (16), (13), (12), (6) (or shortly, (16), (6)).

Again we assume the homogeneous initial condition

$$
\varphi(x, 0)=0, \quad f(x, 0)=0, \quad x \in \bar{D} .
$$

Under condition (17) the following estimate holds for the solution of difference scheme (16),

$$
\left|u(x, t)-z^{(3)}(x, t)\right| \leq M\left[N^{-2} \ln ^{2} N+\tau^{3}\right], \quad(x, t) \in \bar{G}_{h} .
$$

In a similar way we can construct difference schemes with $\varepsilon$-uniform order convergence $\mathcal{O}\left(N^{-2} \ln ^{2} N+\tau^{n}\right), n>3$, i.e. an arbitrary high order of timeaccuracy. 


\section{Numerical results for the time-accurate schemes}

a) In order to illustrate the effect of the defect correction, we consider a singularly perturbed boundary value problem with the Neumann condition in the half-strip. The solution of the problem

$$
\begin{gathered}
L_{(8)} V(x, t)=0, \quad 0<x<\infty, \quad 0<t \leq T, \\
\varepsilon \frac{\partial}{\partial x} V(0, t)=-(128 / 35) \pi^{(-1 / 2)} t^{7 / 2}, \quad 0<t \leq T, \\
V(x, 0)=0, \quad 0 \leq x<\infty,
\end{gathered}
$$

is given by

$$
\begin{aligned}
& V(x, t)=\operatorname{erfc}\left(\frac{x}{2 \varepsilon \sqrt{t}}\right)\left(\frac{x^{8}}{1680 \varepsilon^{8}}+\frac{x^{6}}{30 \varepsilon^{6}} t+\frac{x^{4}}{2 \varepsilon^{4}} t^{2}+\frac{2 x^{2}}{\varepsilon^{2}} t^{3}+t^{4}\right)- \\
& -\frac{1}{\sqrt{\pi}} \exp \left(\frac{-x^{2}}{4 \varepsilon^{2} t}\right)\left(\frac{x^{7}}{840 \varepsilon^{7}} t^{1 / 2}+\frac{9 x^{5}}{140 \varepsilon^{5}} t^{3 / 2}+\frac{37 x^{3}}{42 \varepsilon^{3}} t^{5 / 2}+\frac{93 x}{35 \varepsilon} t^{7 / 2}\right) .
\end{aligned}
$$

Now we study the model problem

$$
\begin{gathered}
L_{(8)} u(x, t)=0, \quad(x, t) \in G, \\
\varepsilon \frac{\partial}{\partial x} u(x, t)=\varepsilon \frac{\partial}{\partial x} V_{(20)}(x, t), \quad(x, t) \in S_{1}, \quad x=0, \\
u(x, t)=V_{(20)}(x, t), \quad(x, t) \in S, \quad x \neq 0 .
\end{gathered}
$$

Then the function $V_{(20)}(x, t)$ is the solution of problem $(21)$.

b) Strictly speaking, the problem (21) is a problem with mixed boundary conditions, that is with Neumann and Dirichlet conditions at the left and the right boundary respectively. The solution has a boundary layer character, and at the point $x=1, V(x, t)$ is exponentially small for $\varepsilon \rightarrow 0$. Therefore, actually problem (21) is a problem with a Neumann condition. We use for the approximation of problem (21) the schemes which are formed for $x<1$ by the mesh equations (12), (6); (13), (6) and (16), (6) and for $x=1$ by the following discrete equations (Dirichlet condition)

$$
z^{(k)}(x, t)=V_{(20)}(x, t), \quad(x, t) \in S, \quad x=1 .
$$

These schemes we denote briefly by (12), (22), (6); (13), (22), (6) and (16), (22), (6) respectively. 
As the solution of boundary value problem (21) has a boundary layer at the left side, for its solution we use the locally condensed mesh

$$
\bar{G}_{h}^{(*)}=\bar{\omega}^{(*)} \times \bar{\omega}_{0}
$$

where $\bar{\omega}^{(*)}=\bar{\omega}^{(*)}(\sigma)$ is a special mesh, condensed in the neighborhood of the left end of the interval $[0,1] ; \sigma$ is the parameter depending on $\varepsilon$ and $N$. The mesh $\bar{\omega}^{(*)}(\sigma)$ is a piecewise constant mesh with constant steps $h_{(1)}$ and $h_{(2)}$ on the intervals $[0, \sigma]$ and $[\sigma, 1], h_{(1)}=\sigma(N / 2)^{-1}, h_{(2)}=(1-\sigma)(N / 2)^{-1}$. We take $\sigma=\min [1 / 2,2 \varepsilon \ln N]$.

According to the theory, the difference schemes (12), (22), (23); (13), (22), (23) and (16), (22), (23) converge respectively with order 1, 2 and 3 w.r.t. $\tau$.

$$
\begin{aligned}
& \left|u(x, t)-z_{(12,22,23)}^{(1)}(x, t)\right| \leq M\left(N^{-2} \ln ^{2} N+\tau\right), \quad(x, t) \in \bar{G}_{h}^{(*)}, \\
& \left|u(x, t)-z_{(13,22,23)}^{(2)}(x, t)\right| \leq M\left(N^{-2} \ln ^{2} N+\tau^{2}\right), \quad(x, t) \in \bar{G}_{h}^{(*)}, \\
& \left|u(x, t)-z_{(16,22,23)}^{(3)}(x, t)\right| \leq M\left(N^{-2} \ln ^{2} N+\tau^{3}\right), \quad(x, t) \in \bar{G}_{h}^{(*)} .
\end{aligned}
$$

To demonstrate this effect numerically, we solve problem (21), using the schemes (12), (22), (23); (13), (22), (23) and (16), (22), (23) for various values of $N, K$ and $\varepsilon$.

c) Results from numerical experiments for the above model Neumann Problem are given in the Tables 2-4 and they are analogous to the results for the Dirichlet problem in Hemker et al ${ }^{6,7}$.

We know that the error of the numerical solution consists of two components related to two contributions: one caused by the discretization of the time derivative and the other by the space derivative. From the theory we know that for the basic scheme the order of convergence is one in $\tau$, and almost two in $h$. This dependence can be easy observed from the error tables, in the regions where one component of error is neglectable w.r.t. the other. Thus, to see the first order of convergence in $\tau$, we should consider the errors where the contribution from the discretization of the space derivative is relatively small. Referring to Tables 2-4, these errors are in the upper-right corner of the table.

It is convenient to introduce the notion of "correct behavior" (w.r.t. the time variable) of the global error if the ratio of the errors for the same $N$ and varying $K$ decreases as the ratio of relative time steps (with a power $p$ if the method is convergent with an order $p$ w.r.t. the time). Using the notion of correct behavior of the global error for Table 2, we see that for $\varepsilon=1$ the 


\begin{tabular}{|c|c|ccccc|}
\hline \multicolumn{2}{|c|}{$N$} & 8 & 32 & 128 & 512 & 2048 \\
\hline$\varepsilon$ & $K$ & & & & & \\
\hline 1 & 8 & $1.01(-1)$ & $1.08(-1)$ & $1.08(-1)$ & $1.08(-1)$ & $1.08(-1)$ \\
& 32 & $2.15(-2)$ & $2.73(-2)$ & $2.78(-2)$ & $2.78(-2)$ & $2.78(-2)$ \\
& 128 & $2.73(-3)$ & $6.53(-3)$ & $6.96(-3)$ & $6.99(-3)$ & $6.99(-3)$ \\
& 512 & $5.94(-3)$ & $1.35(-3)$ & $1.72(-3)$ & $1.75(-3)$ & $1.75(-3)$ \\
& 2048 & $7.26(-3)$ & $1.72(-4)$ & $4.09(-4)$ & $4.36(-4)$ & $4.37(-4)$ \\
\hline $2^{-2}$ & 8 & $6.98(-2)$ & $1.20(-1)$ & $1.26(-1)$ & $1.27(-1)$ & $1.27(-1)$ \\
& 32 & $7.56(-2)$ & $2.51(-2)$ & $3.09(-2)$ & $3.13(-2)$ & $3.14(-2)$ \\
& 128 & $1.01(-1)$ & $3.99(-3)$ & $7.36(-3)$ & $7.79(-3)$ & $7.82(-3)$ \\
& 512 & $1.07(-1)$ & $5.78(-3)$ & $1.56(-3)$ & $1.92(-3)$ & $1.95(-3)$ \\
& 2048 & $1.09(-1)$ & $7.25(-3)$ & $2.49(-4)$ & $4.60(-4)$ & $4.86(-4)$ \\
\hline $2^{-4}$ & 8 & $1.87(-1)$ & $7.76(-2)$ & $1.20(-1)$ & $1.26(-1)$ & $1.27(-1)$ \\
& 32 & $2.91(-1)$ & $5.16(-2)$ & $2.51(-2)$ & $3.09(-2)$ & $3.13(-2)$ \\
& 128 & $3.17(-1)$ & $7.64(-2)$ & $3.99(-3)$ & $7.36(-3)$ & $7.79(-3)$ \\
& 512 & $3.23(-1)$ & $8.26(-2)$ & $5.78(-3)$ & $1.56(-3)$ & $1.92(-3)$ \\
& 2048 & $3.25(-1)$ & $8.42(-2)$ & $7.25(-3)$ & $1.58(-3)$ & $1.61(-3)$ \\
\hline $2^{-6}$ & 8 & $1.87(-1)$ & $7.76(-2)$ & $1.16(-1)$ & $1.26(-1)$ & $1.27(-1)$ \\
$\&$ & 32 & $2.91(-1)$ & $5.16(-2)$ & $2.30(-2)$ & $3.02(-2)$ & $3.13(-2)$ \\
$2^{-8}$ & 128 & $3.17(-1)$ & $7.64(-2)$ & $3.46(-3)$ & $6.79(-3)$ & $7.71(-3)$ \\
& 512 & $3.23(-1)$ & $8.26(-2)$ & $9.38(-3)$ & $1.52(-3)$ & $1.85(-3)$ \\
& 2048 & $3.25(-1)$ & $8.42(-2)$ & $1.09(-2)$ & $1.55(-3)$ & $1.59(-3)$ \\
\hline
\end{tabular}

Table 2: Table of errors $E(N, K, \varepsilon)$ for scheme (12), (22) $E(N, K, \varepsilon)$ is defined by $(9)$, where $z(x, t)=z_{(12,22,23)}^{(1)}(x, t), u^{*}(x, t)=V_{(20)}(x, t)$,

$$
\bar{G}_{h}=\bar{G}_{h(23)}^{(*)} \text {. }
$$

domain with correct behavior of the error is the major part of the table, related to $\varepsilon=1$ and laying on the upper-right side.

As the value of $\varepsilon$ decreases, the domain of correct behavior of the error tends to decrease w.r.t. that domain for $\varepsilon=1$. This can be explained by the relative increasing influence of the space error for smaller $\varepsilon$. In the case of $\varepsilon \leq$ $2^{-6}$ the domain of correct behavior of the error does not longer change. Thus, in Table 2 we can observe the $\varepsilon$-uniform convergence, of order (approximately) one w.r.t. the time.

Now we analyze the analogous tables, which give the errors for $z^{(2)}(x, t)$ and $z^{(3)}(x, t)$ that are the corrected solutions. Note that, in principle, the time correction does not improve the space-accuracy. By the correction we improve only the time component in the error, and we observe the improvement only when the space-dependent contribution of the error is small in comparison with the time-dependent contribution.

In order to check the expected second and third order time-accuracy respectively in Tables 3 for $z^{(2)}(x, t)$ and a corresponding table for $z^{(3)}(x, t)$ (not shown by lack of space), we should compare the ratios of the errors for fixed 


\begin{tabular}{|c|c|ccccc|}
\hline \multicolumn{2}{|c|}{$N$} & 8 & 32 & 128 & 512 & 2048 \\
\cline { 1 - 5 }$\varepsilon$ & $K$ & & & & & \\
\hline 1 & 8 & $2.94(-2)$ & $2.01(-2)$ & $1.63(-2)$ & $1.53(-2)$ & $1.51(-2)$ \\
& 32 & $1.11(-3)$ & $2.13(-3)$ & $1.38(-3)$ & $1.11(-3)$ & $1.03(-3)$ \\
& 128 & $5.98(-3)$ & $7.75(-5)$ & $1.38(-4)$ & $8.84(-5)$ & $7.06(-5)$ \\
& 512 & $7.27(-3)$ & $3.76(-4)$ & $4.91(-6)$ & $8.71(-6)$ & $5.55(-6)$ \\
& 2048 & $7.59(-3)$ & $4.58(-4)$ & $2.35(-5)$ & $2.83(-7)$ & $5.94(-7)$ \\
\hline $2^{-2}$ & 8 & $1.75(-2)$ & $2.92(-2)$ & $1.85(-2)$ & $1.45(-2)$ & $1.34(-2)$ \\
& 32 & $8.49(-2)$ & $1.41(-3)$ & $2.05(-3)$ & $1.24(-3)$ & $9.47(-4)$ \\
& 128 & $1.03(-1)$ & $5.96(-3)$ & $9.27(-3)$ & $1.32(-4)$ & $7.86(-5)$ \\
& 512 & $1.08(-1)$ & $7.31(-3)$ & $3.75(-4)$ & $9.92(-6)$ & $1.02(-5)$ \\
& 2048 & $1.09(-1)$ & $7.64(-3)$ & $4.60(-4)$ & $2.34(-5)$ & $1.02(-5)$ \\
\hline $2^{-4}$ & 8 & $1.95(-1)$ & $1.98(-2)$ & $2.92(-2)$ & $1.85(-2)$ & $1.45(-2)$ \\
& 32 & $2.93(-1)$ & $6.28(-2)$ & $1.55(-3)$ & $2.05(-3)$ & $1.58(-3)$ \\
& 128 & $3.17(-1)$ & $7.92(-2)$ & $5.96(-3)$ & $1.55(-3)$ & $1.58(-3)$ \\
& 512 & $3.23(-1)$ & $8.33(-2)$ & $7.31(-3)$ & $1.55(-3)$ & $1.58(-3)$ \\
& 2048 & $3.25(-1)$ & $8.43(-2)$ & $7.64(-3)$ & $1.58(-3)$ & $1.61(-3)$ \\
\hline $2^{-6}$ & 8 & $1.95(-1)$ & $1.98(-2)$ & $3.06(-2)$ & $2.12(-2)$ & $1.58(-2)$ \\
$\&$ & 32 & $2.93(-1)$ & $6.28(-2)$ & $2.39(-3)$ & $2.30(-3)$ & $1.59(-3)$ \\
$2^{-8}$ & 128 & $3.17(-1)$ & $7.92(-2)$ & $9.21(-3)$ & $1.52(-3)$ & $1.59(-3)$ \\
& 512 & $3.23(-1)$ & $8.33(-2)$ & $1.08(-2)$ & $1.52(-3)$ & $1.59(-3)$ \\
& 2048 & $3.25(-1)$ & $8.43(-2)$ & $1.12(-2)$ & $1.55(-3)$ & $1.59(-3)$ \\
\hline
\end{tabular}

Table 3: Table of Errors $E(N, K, \varepsilon)$ for scheme (13), (22) $E(N, K, \varepsilon)$ is defined by (9), where $z(x, t)=z_{(13,22,23)}^{(2)}(x, t), u^{*}(x, t)=V_{(20)}(x, t)$,

$$
\bar{G}_{h}=\bar{G}_{h(23)}^{(*)} \text {. }
$$

$N$ and varying $K$, and the ratios of related time steps squared in Table 3 and to the 3rd power in the table not shown.

Analyzing Table 3 , we see that the domain with correct behavior of the error is smaller than in Table 2 and it decreases when the parameter $\varepsilon$ decreases. Note that, for small $\varepsilon$, the part of Table 3 with correct behavior of the error is much reduced because the portion of the space error is relatively large for the corrected solution. Again, for $\varepsilon \leq 2^{-6}$ the errors for the same $N, K$ do not change. Consequently, we see an $\varepsilon$-uniform effect of the improvement of the accuracy. The order of convergence w.r.t. $\tau$ is about two.

Because the space error is smaller for $\varepsilon=1$, this case shows more clearly the effect of the defect correction in time. For illustration, Table 4 shows the convergence order, defined as $P=\log _{2}\left(E\left(N_{i}, K_{j}, 1\right) / E\left(N_{i}, K_{j+1}, 1\right)\right)$. For the construction of Table 4 the same data are used as for the Tables 2 and 3 , and the "incorrect behavior" of the error is indicated by *. We can see that, in practice, the order of convergence corresponds with the theoretical result.

Comparing the Tables 2, 3, and the corresponding table for order 3 , we summarize the observations: (i) already at $K=32$ (for all $\varepsilon$ and $N$ ) the error 


\begin{tabular}{|c|c|ccccccccc|}
\hline \multicolumn{2}{|c|}{$N$} & 8 & 16 & 32 & 64 & 128 & 256 & 512 & 1024 & 2048 \\
\cline { 1 - 8 }$n$ & $K$ & & & & & & & & & \\
\hline 1 & 8 & 1.07 & 1.00 & .98 & .98 & .98 & .98 & .98 & .98 & .98 \\
& 16 & 1.16 & 1.04 & 1.00 & .99 & .99 & .99 & .99 & .99 & .99 \\
& 32 & 1.33 & 1.09 & 1.02 & 1.00 & 1.00 & .99 & .99 & .99 & .99 \\
& 64 & 1.64 & 1.18 & 1.05 & 1.01 & 1.00 & 1.00 & 1.00 & 1.00 & 1.00 \\
& 128 & $*$ & 1.34 & 1.09 & 1.02 & 1.00 & 1.00 & 1.00 & 1.00 & 1.00 \\
& 256 & $*$ & 1.63 & 1.18 & 1.05 & 1.01 & 1.00 & 1.00 & 1.00 & 1.00 \\
& 512 & $*$ & $*$ & 1.34 & 1.09 & 1.02 & 1.01 & 1.00 & 1.00 & 1.00 \\
& 1024 & $*$ & $*$ & 1.63 & 1.18 & 1.05 & 1.01 & 1.00 & 1.00 & 1.00 \\
\hline 2 & 8 & 1.80 & 1.57 & 1.62 & 1.72 & 1.81 & 1.86 & 1.89 & 1.91 & 1.92 \\
& 16 & 2.92 & 1.83 & 1.61 & 1.66 & 1.76 & 1.84 & 1.90 & 1.93 & 1.95 \\
& 32 & $*$ & 2.93 & 1.84 & 1.63 & 1.68 & 1.78 & 1.86 & 1.92 & 1.95 \\
& 64 & $*$ & $*$ & 2.94 & 1.85 & 1.64 & 1.69 & 1.79 & 1.87 & 1.92 \\
& 128 & $*$ & $*$ & $*$ & 2.95 & 1.85 & 1.65 & 1.69 & 1.79 & 1.87 \\
& 256 & $*$ & $*$ & $*$ & $*$ & 2.96 & 1.85 & 1.65 & 1.70 & 1.79 \\
& 512 & $*$ & $*$ & $*$ & $*$ & $*$ & 3.01 & 1.85 & 1.65 & 1.69 \\
& 1024 & $*$ & $*$ & $*$ & $*$ & $*$ & $*$ & 3.10 & 1.72 & 1.54 \\
\hline 3 & 8 & $*$ & 1.79 & 3.73 & 3.19 & 3.02 & 3.00 & 3.01 & 3.02 & 3.02 \\
& 16 & $*$ & $*$ & $*$ & 2.67 & 3.52 & 3.10 & 2.99 & 2.98 & 2.98 \\
& 32 & $*$ & $*$ & $*$ & $*$ & $*$ & 4.03 & 3.30 & 3.03 & 2.98 \\
& 64 & $*$ & $*$ & $*$ & $*$ & $*$ & $*$ & 1.84 & 3.65 & 2.99 \\
& 128 & $*$ & $*$ & $*$ & $*$ & $*$ & $*$ & $*$ & $*$ & 2.99 \\
\hline
\end{tabular}

Table 4: Table of Convergence Orders under $\varepsilon=1$ for corresponding $z^{(k)}(x, t), \quad k=1,2,3$.

for $z^{(3)}(x, t)$ is practically equal to the space component and it is smaller than for $z^{(1)}(x, t)$ at $K=2048$; (ii) the order of $\varepsilon$-uniform convergence w.r.t. $\tau$ is better for scheme (13), (22) than that for scheme (12), (22), and the order for scheme (16), (22) is better than for scheme (13), (22); (iii) the order of convergence w.r.t. $\tau$ increases for the functions $z^{(k)}(x, t)$ for increasing $k$; (iv) the order of convergence w.r.t. the space variable is nearly two, $\varepsilon$-uniform for sufficiently large $N$.

Thus, numerical results confirm the theoretical results and demonstrate the efficiency of the defect correction.

\section{Conclusion}

In this paper we show the feasibility of a defect correction procedure, that can easily be implemented in order to improve the time-accuracy in a parabolic $\mathrm{PDE}$, for an $\varepsilon$-uniformly convergent discretization scheme in the case of a Neumann Problem. In this way the order of accuracy w.r.t. the time and the space variables can be made of the same order of magnitude.

The defect correction process improves only the accuracy w.r.t. the time discretization and does not change the approximation w.r.t. the space dis- 
cretization. Therefore, by application of the defect correction, the principal part of the total error becomes the part due to the approximation of the space derivatives even for relatively small values of $K$, if the higher order corrections are applied.

By defect correction we are able to increase the accuracy of the approximate solution essentially, viz. from 1st to 2 nd and 3 rd order in $\tau$. For this we use the same time grid for the accurate and for the auxiliary solutions.

In addition, numerical results illustrate that, also in practice, for the special piecewise uniform grid, the order of convergence w.r.t. the space variable is close to two.

\section{Acknowledgments}

This research was supported in part by the Dutch Research Organization NWO under grant N 047.003.017 and by the Russian Foundation for Basic Research under grant N 98-01-00362.

\section{References}

1. P.A. Farrell, P.W. Hemker, and G.I. Shishkin, Journal of Computational Mathematics 14, 71 (1996).

2. P.A. Farrell, P.W. Hemker, and G.I. Shishinin, J. Comp. Math. 14, 273 (1996).

3. P.A. Farrell, J.J.H. Miller, E. O'Riordan and G.I. Shishkin, SIAM Journal on Numerical Analysis 33, 1135 (1996).

4. G.I.Shishkin, Mesh Approximation of Singularly Perturbed Elliptic and Parabolic Equations (Ur. O. RAN, Ekaterinburg, 1992) (in Russian).

5. J.J.H. Miller, E. O'Riordan, G.I. Shishkin, Fitted numerical methods for singularly perturbation problems (World Scientific, Singapore, 1996).

6. P.W. Hemker, G.I. Shishkin and L.P. Shishkina, ZAMM 59, (1997).

7. P.W. Hemker, G.I. Shishkin and L.P. Shishkina, IMA 14, 1 (1998). 